\title{
THE ROLE OF MAGNETIC RECONNECTION IN FLARES AND PROMINENCE ERUPTIONS
}

\author{
T.G. FORBES \\ Institute for the Study of Earth, Oceans, and Space \\ University of New Hampshire \\ Durham, NH 03824, USA
}

\begin{abstract}
Magnetic Reconnection is often invoked as the primary mechanism for driving a flare or a prominence eruption. Here I argue that a catastrophic loss of mechanical equilibrium, rather than reconnection, is probably the primary mechanism for driving these phenomena. However, reconnection is still essential in order for any significant amount of energy to be released. To illustrate this idea we present some recent results from an MHD simulation based on a catastrophe mechanism first proposed by Van Tend and Kuperus. In order for this mechanism to be effective a substantial amount of reconnection must occur within a few Alfvén-scale times. Such rapid reconnection is plausible since the loss of mechanical equilibrium can generate flows which drive the reconnection at a rapid rate.
\end{abstract}

\section{Introduction}

It is often stated that magnetic reconnection causes flares and prominence eruptions. However, I would like to suggest that reconnection is not the basic mechanism behind these phenomena. This does not necessarily mean that reconnection is unimportant, since in the scenario that I will discuss in this talk, reconnection is still an essential process without which two-ribbon flares and prominence eruptions would be impossible.

My present view of how reconnection works in these eruptive phenomena is based on recent analytical and numerical MHD models developed by myself and E.R. Priest. Our models were inspired by the early circuit models of Van Tend and Kuperus (1978) and Kaastra (1985) which treat the prominence, or pre-flare filament, as a line current suspended above the photosphere (see also Martens and Kuin, 1989). The basic magnetic mechanism which drives the current filament upwards in these models is illustrated in Figure 1. In Figure 1a, a current filament (shaded region) is stably suspended above the photosphere (hatched region) because the field lines surrounding the current filament are line-tied to the photosphere. If the filament is perturbed, it simply oscillates up and down around its equilibrium location as indicated in the energy diagram in Figure $1 \mathrm{~b}$. However, this stable equilibrium vanishes if the field lines surrounding the filament are disconnected from the photosphere so that the magnetic configuration approaches that shown in Figure 1c. As the energy diagram in Figure 1d indicates, the only stable equilibrium location for the configuration shown in Figure 1c occurs at infinite height.

Van Tend (1979) showed that a transition like that shown in Figure 1 can occur either continuously or discontinuously as the strength of the background field relative to the current in the 
filament is decreased. In the purely two-dimensional system considered by Van Tend, the transition is discontinuous only if the photospheric background field falls off with height faster than $1 / y$, where $y$ is the vertical coordinate. In order for the field to decrease with height in this fashion, the photospheric background field must have a two-dimensional dipole, or higher order

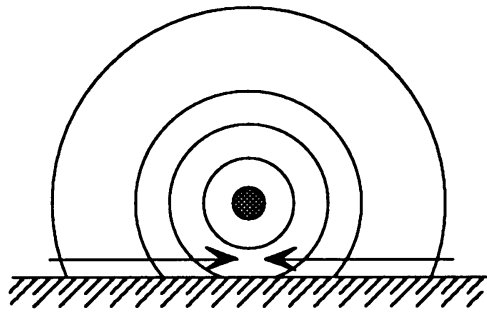

(a)

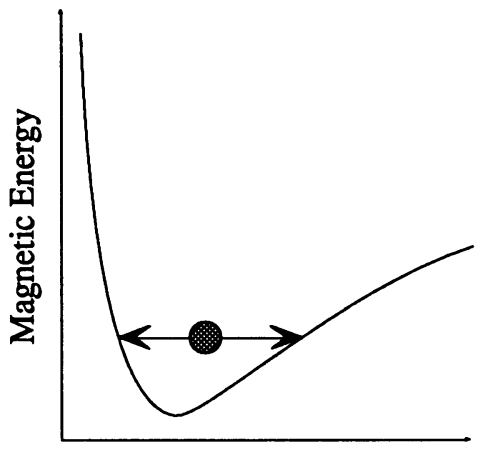

Height

(b)

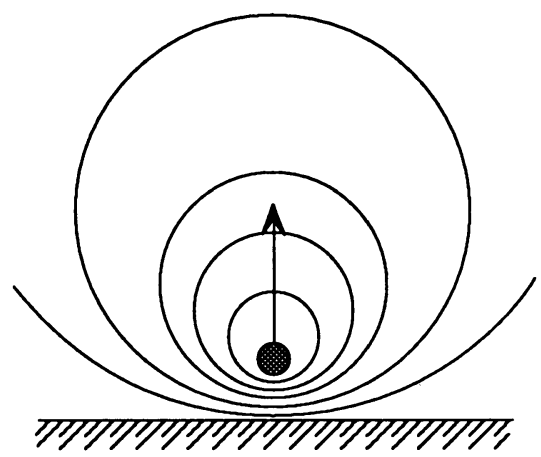

(c)

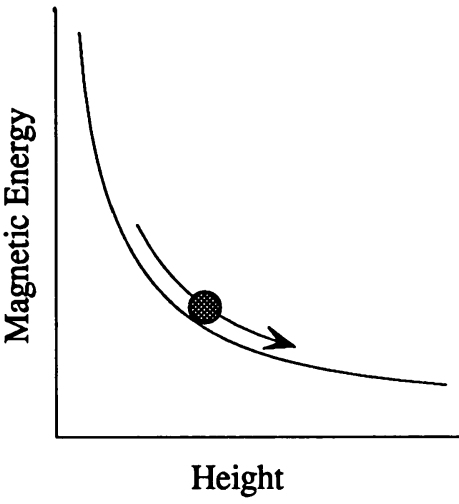

(d)

Figure 1. Schematic diagram of the driving mechanism in the Van Tend and Kuperus (1978) model. The shaded circle designates the current filament.

multipole, component. If the background field has only a two-dimensional monopole component (i.e. a line-current), than there is no sudden transition in filament height as the relative strength of the background field weakens.

In the original analysis by Van Tend and Kuperus (1978), the current filament was simply treated as a wire immersed in a vacuum, so that the magnetic field lines were not frozen to a plasma as they are in ideal MHD. Consequently, in their model, reconnection occurs freely. However, in a realistic plasma environment any attempt to change rapidly a configuration with an $x$-line in it leads to the formation of a current sheet at the $\mathrm{x}$-line since reconnection is inhibited by the conductivity of the plasma. Kaastra (1985) and Martens and Kuin (1989) attempted to address this problem by 
incorporating current sheets within the circuit framework of Van Tend and Kuperus, but from an MHD point of view neither Kaastra's nor Martens and Kuin's model is self-consistent. Both models use Syrovatskii's (1971) solution for a current sheet to prescribe the properties of the current sheet. Although Syrovatskii's solution is a valid solution of the ideal MHD equations in the limit of low plasma $\beta$ (i.e. strong magnetic fields), it has boundary conditions at infinity which are not consistent with the Van Tend and Kuperus model.

\section{An Analytical MHD Model}

Recently, E.R. Priest and myself developed a relatively simple Van-Tendand-Kuperus-type model which is a self-consistent solution of the idealMHD equations in the limit of low plasma $\beta$ (Priest and Forbes, 1990). In complex notation the magnetic field of this model is

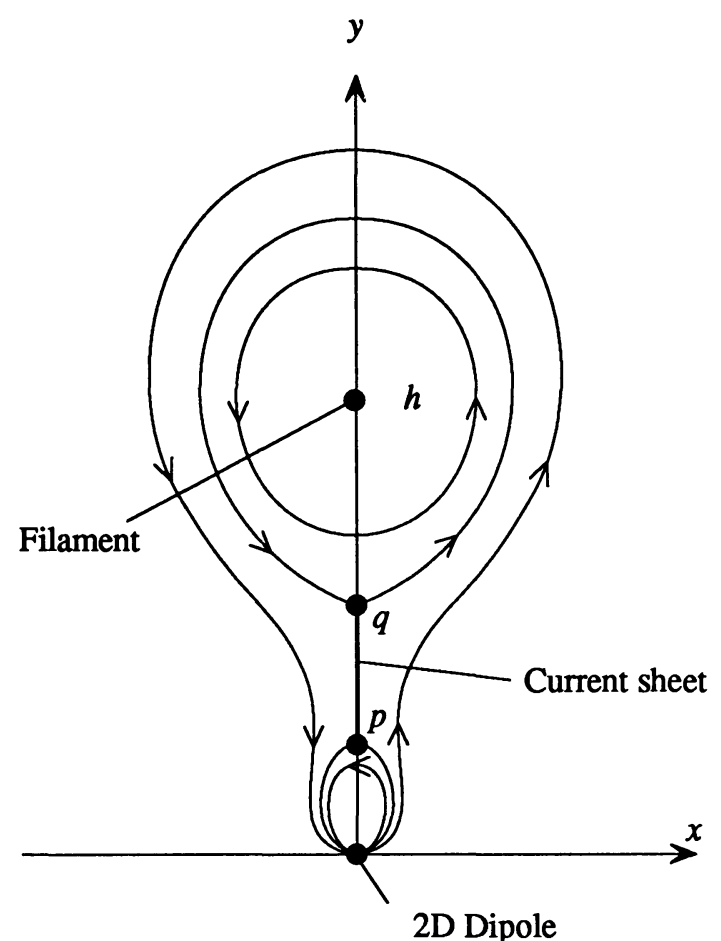

Figure 2. Magnetic field of the analytical MHD model .

$$
B_{y}+i B_{x}=i \frac{m h^{2} \sqrt{\left(z^{2}+p^{2}\right)\left(z^{2}+q^{2}\right)}}{p q z^{2}\left(z^{2}+h^{2}\right)}
$$

where $z=x+i y, h$ is the height of the filament, and $p$ and $q$ are the upper and lower end points of an infinitely thin current sheet located on the imaginary axis as shown in Figure 2. The quantity $m$ is the strength of a dipole at $z=0$ and this dipole represents the photospheric background field. Application of the frozen-flux condition gives the relation:

$$
2 h p q \sqrt{\left(h^{2}-p^{2}\right)\left(h^{2}-q^{2}\right)}=m / I(h)
$$

where $I(h)$ is the filament current. In the limit that the radius of the filament current tends to zero, $I(h)$ becomes constant.

When the background dipole is placed at a depth, $h_{b}$, below the photosphere, equilibria occur at

$$
h=m / I-h_{b} \pm\left[(m / I)^{2}-2 h_{b}(m / I)\right]^{1 / 2}
$$

as shown in Figure 3 for $h_{b}=1$. This set of equilibria has both stable and unstable branches, but 
there are no equilibria when $m$ is less than the critical value of $2 I h_{b}$. As $h_{b}$ tends to zero, the unstable branch approaches $h=2 \mathrm{~m} / \mathrm{I}$, while the stable branch approaches $h=0$.

Figure 4 compares the energy curve of this ideal-MHD model with no reconnection) to the energy

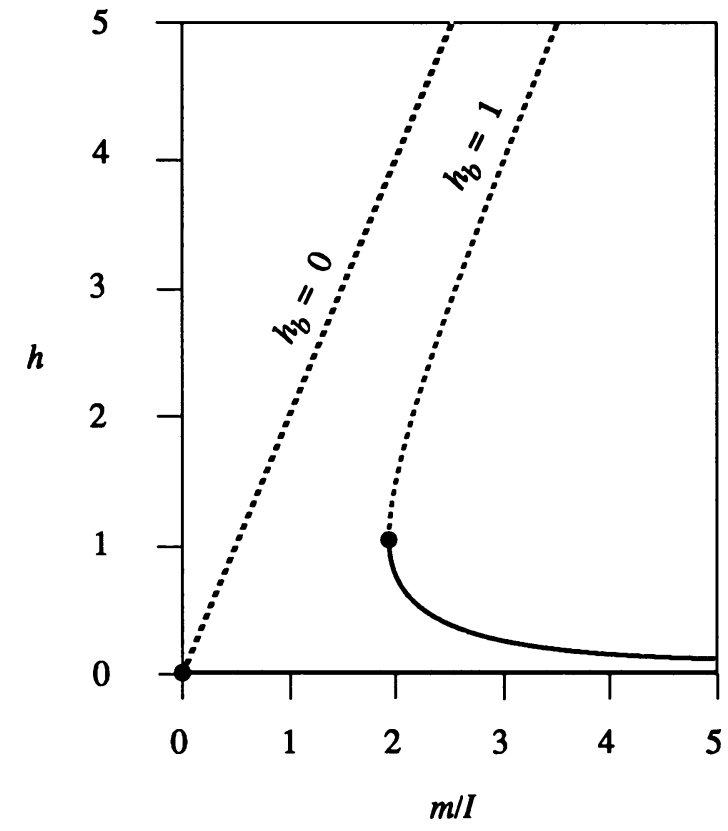

Figure 3. Equilibrium filament heights as a function of the ratio of the relative dipole strength. The dashed curves denote unstable equilibria, and $h_{b}$ is the dipole depth below the surface (arbitrary units).

$$
\begin{aligned}
& \partial \mathbf{B} / \partial t=-\nabla \times(\mathbf{v} \times \mathbf{B})+\eta \nabla^{2} \mathbf{B} \\
& \partial \rho / \partial t=-\nabla \cdot(\rho \mathbf{v}) \\
& \rho[\partial \mathbf{v} / \partial t+(\mathbf{v} \cdot \nabla) \mathbf{v}]=-\nabla p+\mathbf{j} \times \mathbf{B}+\rho v \nabla^{2} \mathbf{v} \\
& \rho c_{v} T(\partial / \partial t+\mathbf{v} \cdot \nabla) \log (p / \rho \gamma)=\eta j^{2}+\rho v(\nabla \cdot \mathbf{v})^{2} \\
& p=R \rho T
\end{aligned}
$$
curve of a comparable Van Tend and Kuperus model with uninhibited reconnection. In both models the magnetic energy of the system decreases with increasing height. Thus despite the formation of a current sheet in the MHD case, the filament still moves upwards. However, in the absence of reconnection the energy release is only $12.3 \%$ of that occurring when the reconnection is uninhibited. The remaining $87.7 \%$ of the available magnetic energy is stored in the field produced by the current sheet, and this energy can only be released by reconnection.

\section{A Numerical MHD Model}

Although Van Tend and Kuperus's model was originally discussed within the framework of circuit theory, it is possible to formulate the model as an initial-boundary-value problem for the time-dependent MHD equations. To illustrate how this can be done, consider the following system of equations:

in the domain $-\infty<x<\infty, 0<y \infty$. For this system the dependent variables are the magnetic field, $B$, the velocity, $v$, the density, $\rho$, and the pressure, $p$. The constants $\eta, v, c_{v}, \gamma$, and $R$ are the electrical resistivity, the viscosity, the specific heat at constant volume, the ratio of specific heats (= $5 / 3)$, and the universal gas constant $\left(=2 c_{v} / 3\right)$, respectively. For the numerical simulation $v$ and $\eta$ are set to the smallest possible values which, with a grid mesh of $100 \times 100$ points, gives viscous and magnetic Reynolds numbers of about 200 each. 
The initial magnetic field is the sum of a dipole background at depth $h_{b}$, a current filament with the field

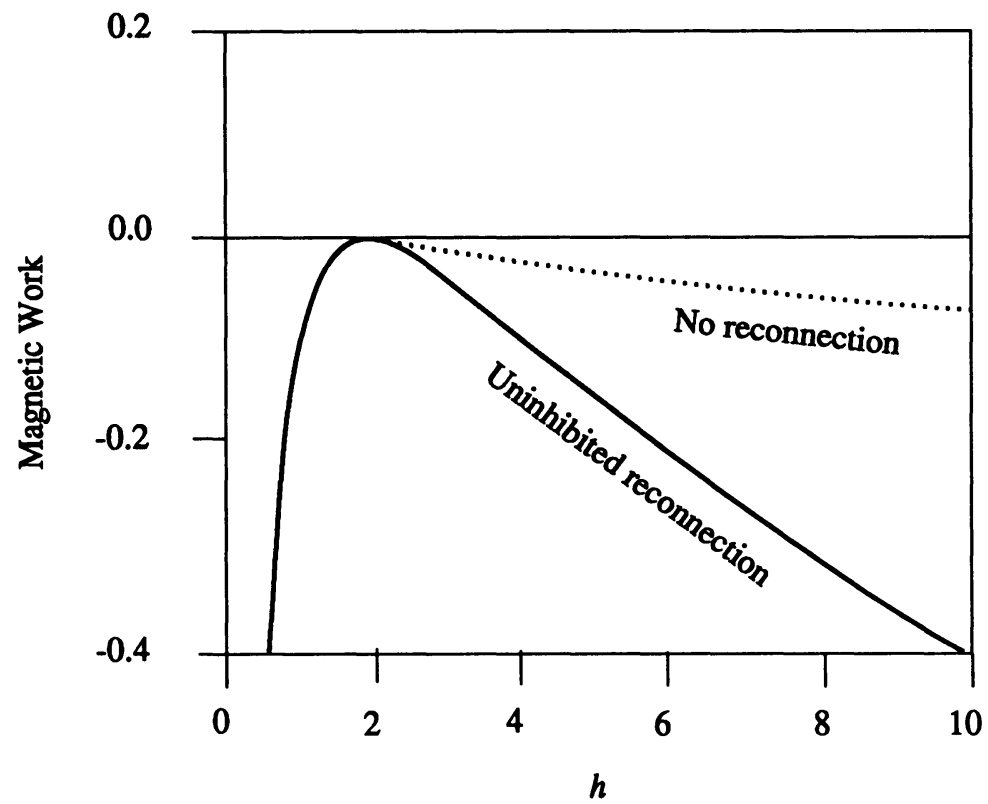

Figure 4. Magnetic work as a function of filament height, $h$, for the MHD (dashed curve) and non-MHD (solid curve) models. In both models $h_{b}=0$.

$$
B_{\phi}= \begin{cases}B_{o}\left(r / r_{o}\right), & r \leq r_{o} \\ B_{o}(r d r), & r \geq r_{o}\end{cases}
$$

centered at $y=h$, and a corresponding image filament at $y=-h$. Here $r_{o}$, the radius of the filament, is set at $\mathbf{0 . 0 5}$ times the half-with of the numerical domain, and initially the plasma is at rest and has a uniform entropy. The initial pressure distribution inside the filament is determined from the requirement that $\nabla p=\mathbf{j} \times \mathbf{B}$, while the pressure outside the filament is uniform (see Forbes 1990 for details).

The boundary conditions at the photosphere $(y=0)$ are:

$$
\begin{array}{ll}
j=0 & \text { (Line-Tying) } \\
v_{x}(x, 0, t)=0 & \text { (No-Slip) } \\
v_{y}(x, 0, t)=0 & \text { (Wall) }
\end{array}
$$

The line-tying condition, $j=0$, follows from the requirement that field lines be anchored to the base. In two-dimensional MHD, field lines correspond to contours of constant $A$, where $A$ is the magnitude of the vector potential. Thus Faraday's equation combined with Ohm's law gives 


$$
E=-\partial A / \partial t=-(\mathbf{v} \times \mathbf{B})_{2}+\eta j=0
$$

Since the wall and no-slip boundary conditions give $v=0$ at the base, $j$ is necessarily zero.

In general this definition of line-tying is not equivalent to the frozen-flux condition. In MHD the field line velocity, $\mathbf{v}_{\mathbf{b}}$, is

$$
\mathbf{v}_{\mathbf{b}}=\mathbf{v}+\eta(\mathbf{J} \times \mathbf{B}) / B^{2} .
$$

and line tying occurs at the base when $\mathbf{v}_{\mathbf{b}}=0$, while frozen flux occurs when $\mathbf{v}_{\mathbf{b}}=\mathbf{v}$. Only for the special case when $\mathbf{v}=0$ are line tying and frozen flux equivalent.

The MHD system of equations (4) - (8) is solved by using the explicit, flux-corrected transport code SHASTA (SHarp And Smooth Transport Algorithm) of Weber et al. (1979). SHASTA is well-suited to studying shock waves since it can sustain a sharp shock transition over only two or

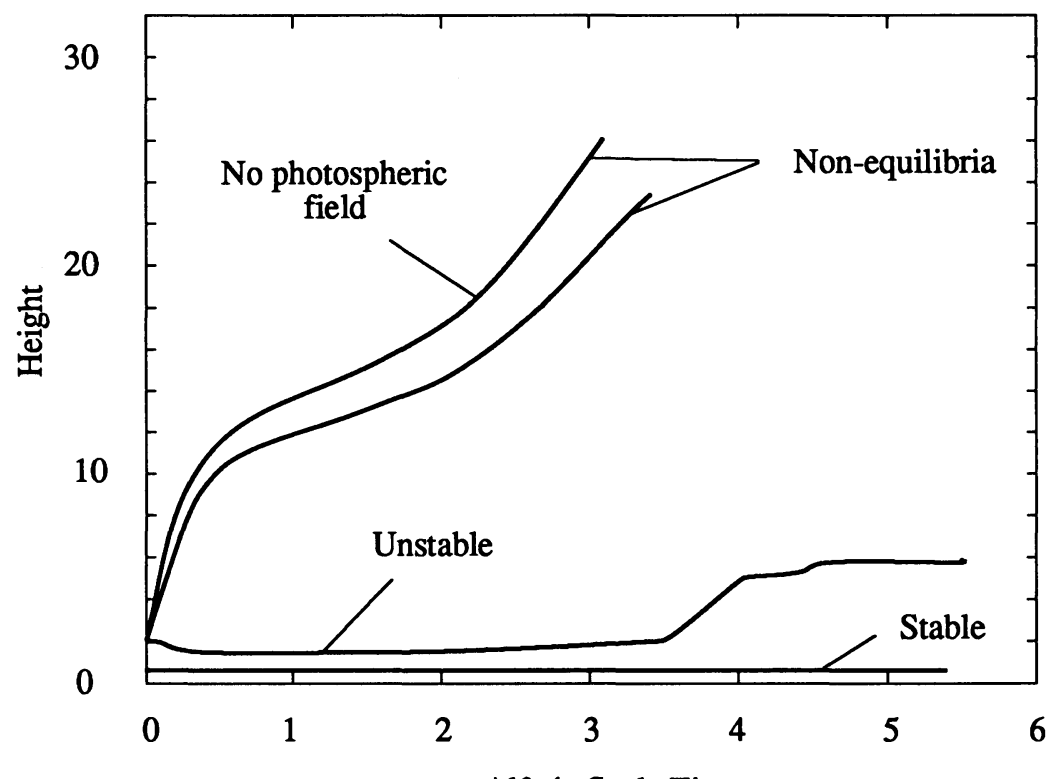

Alfvén Scale Time

Figure 5. Filament height as a function of time with the height in units of $h_{b}$

three mesh points.

One can test the equilibrium properties of the system by varying the filament's height and current relative to the background dipole field. Figure 5 shows the filament height as a function of time for 4 cases all of which start at $h=2 h_{b}$ except for the case labeled 'stable' which starts at $h=0.25 h_{b}$. The nonequilibrium case labeled 'no photospheric field' is for $m=0$, while the other nonequilibrium case is for $m=0.5 h_{b} / I$. The case labeled 'unstable' has $m=2.25 h_{b} / I$, and corresponds to a case which is initially on the unstable branch of the equilibrium curve shown in Figure 3.

As $m$ increases, the background field becomes stronger, and the filament's upward motion becomes increasingly constrained by the tension that develops in the line-tied field lines as they are 
stretched upwards. When $m=2.25 h_{b} / l$ is reached, there is no initial force on the current filament, and the filament is in equilibrium. However, this equilibrium is unstable, and the filament moves upwards until a new equilibrium is reached at about $h=8.1 h_{b}$.

The final height which the filament reaches depends strongly upon the initial radius, $r_{o}$, of the filament. As $r_{o}$ tends to zero the final equilibrium height tends to infinity because the magnetic energy available to drive the filament upwards increases without limit. The final equilibrium height in the analytical model increases only as $1 / \log \left(r_{o}\right)$ ( $c f$. Figure 4$)$, and decreasing the radius by even several orders of magnitude causes only a modest increase in the final equilibrium height. Thus in the absence of significant reconnection, the numerical and analytical solutions together suggest that

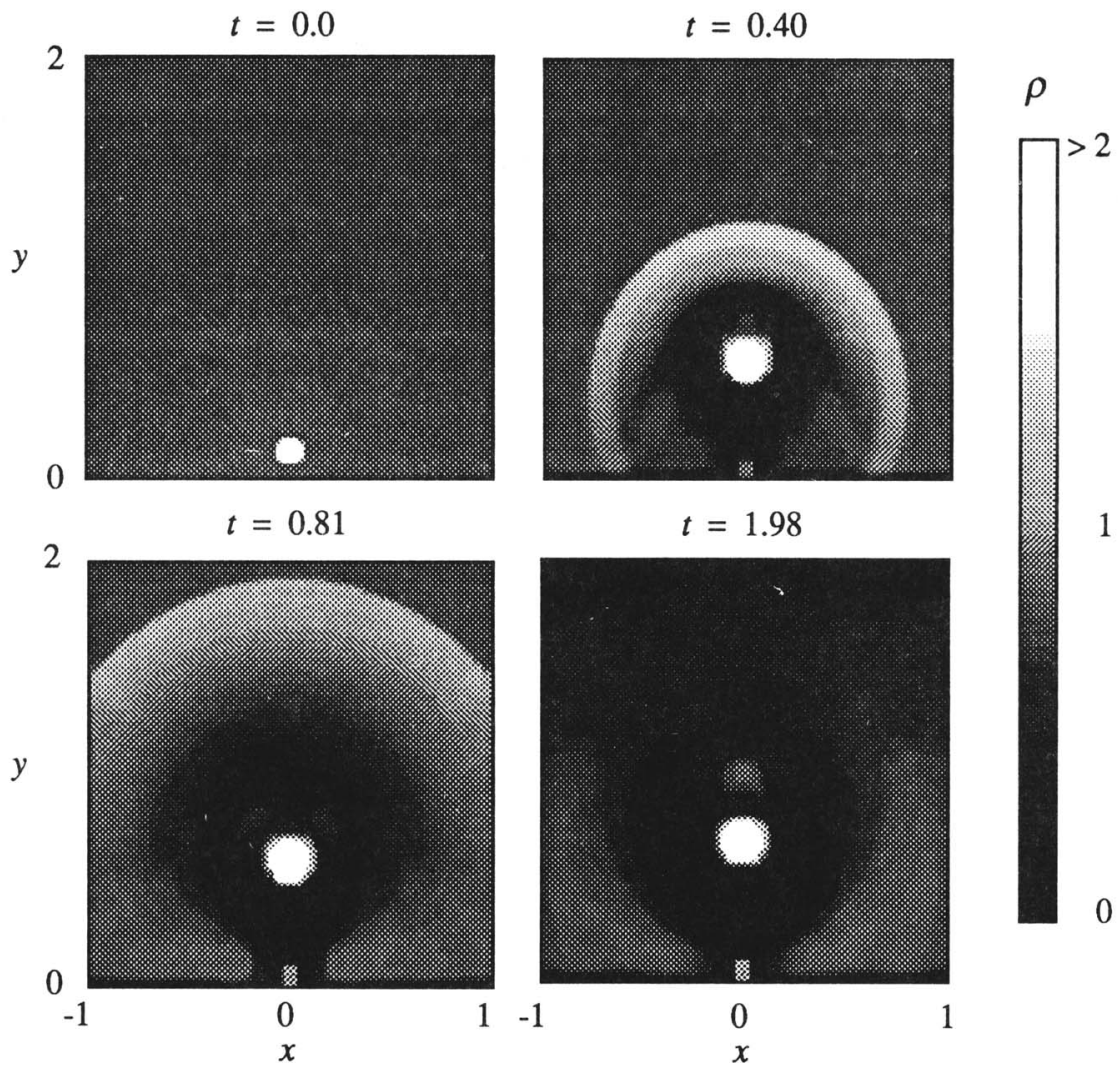

Figure 6. Grey-scale contours of the mass density at 6 different times for the nonequilibrium case with $m=0.5 h_{b} / I$. The filament moves upwards at about 0.3 times the Alfvén speed and is preceded by a fast-mode shock. Times are shown in units of the Alfvén scale-time, and $\rho=1$ corresponds to the ambient coronal density.

the filament is not likely to rise more than roughly 10 times its initial height for any realistic value of the filament radius, $r_{o}$. 


\section{Conclusion}

Using analytical and numerical MHD equivalents of Van Tend and Kuperus's circuit model, I have argued that a catastrophic loss of ideal MHD equilibrium, rather than reconnection, is the underlying mechanism which drives two-ribbon flares, and prominence eruptions. All of the models are based on current filaments suspended in the corona by a balance between magnetic compression and magnetic tension. The compression of field lines trapped between the photosphere and the filament pushes the filament upwards, while the tension of field lines tied to the photosphere and passing over the filament pulls the filament downwards ( $c f$. Figure 1). MHD equilibrium is lost when the strength of the photospheric field relative to the filament field decreases below a critical value.

Although reconnection is not the underlying mechanism which drives the filament upwards, it is still very essential since without it the current filament cannot escape from the lower corona. Furthermore, in order for the current filament to escape, the reconnection must occur rapidly - on the order of the Alfvén scale-time of the system. Such an extraordinarily rapid rate of reconnection may just be possible since the flows produced by the loss of ideal equilibrium act to drive field lines towards each other ( $c f$. Forbes 1990).

During the last few years, studies by Aly (1988) and Klimchuk and Sturrock (1989) have found that several previously proposed catastrophe models for two-ribbons flares and prominence eruptions are not plausible because they require boundary conditions that are not physically acceptable (e.g. Barnes and Sturrock, 1972; Low, 1977; and Bim et al., 1978). At the present time, I believe that the MHD catastrophe model I have presented here does have physically acceptable boundary conditions. However, until a fully three-dimensional version of the model is available, the possibility will remain that the boundary conditions are not physically acceptable.

Acknowledgements. My special thanks go to Vinod Krishan for her efforts in making my trip to this symposium possible. This work was supported by NASA Grant NAGW-76 and the local organizing committee of IAU Symposium 142.

\section{References}

Aly, J.J. (1988) Astron. Astrophys., 203, 183.

Barnes, C.W. and Sturrock, P.A. (1972) Astrophys. J., 174, 659, 1972.

Bim, J., Goldstein, H., and Schindler, K. (1978) Solar Phys. 57, 81.

Forbes, T.G. (1990) J. Geophys. Res., in press.

Kaastra, J.J. (1985) Solar Flares: An Electrodynamics Model, PhD. Thesis, Rijksuniversiteit, Utrecht.

Klimchuk, J.A. and Sturrock, P.A. (1989), Astrophys. J., in press.

Low, B.C. (1977) Astrophys. J., 212, 234.

Martens, P.C.H. and Kuin, N.P.M. (1989) Solar Phys., 22, 263.

Priest, E.R. and Forbes, T.G. (1990) Solar Phys., in press.

Syrovatskii, S.I. (1971) Soviet Phys. JETP, 33, 933.

Van Tend, W. (1979) Solar Phys., 61, 89.

Van Tend, W., and Kuperus, M. (1978) Solar Phys., 59, 115.

Weber, W.J., Boris, J.P., and Gardener, J.H. (1979) Computer Phys. Comm., 16, 243, 1979. 


\section{DISCUSSION}

KUIJPERS: In Kaastra's work the position of the curret sheet is not arbitrary. In fact his self-consistent electrodynamical calculation gives an energy dissipation rate into particle acceleration in agreement with the observations. However he used as an input to the model the observed rise-speed of the filament. Of course in your work it is an important step forward that you can derive the filament speed.

FORBES: I cannot agree that Kaastra's current was calculated self-consistently since he used a current sheet solution of Syrovatsky and just added this current to the other currents. Since Syrovatsky's solution is for much different boundary conditions, Kaastra's solution is not a self-consistent solution of the MHD equations. However, I would still like to emphasize that Kaastra's solution was very worthwhile as a first attempt to construct a quantitative model based on the Van Tend-Kuperus mechanism.

KUNDU: You have discussed the question of particle acceleration in fast CME's. As you know Hundhausen et al at HAO have observed many CME's with slow speeds (50$200 \mathrm{~km} / \mathrm{s}$ ). Everytime we have overlapping radio observations at meter-decameter wavelengths, we find radio emission in the form of type IV and/or type II bursts. Obviously particle acceleration is involved. Have you given any thinking as to how slowmode shocks can accelerate particles responsible for the observed radio emission?

FORBES: I myself have not looked at the possibility of energetic particle acceleration at slow-mode shocks, but Marty Lee and Phil Isenberg in our group at the University of New Hampshire have done so. In their opinion there is, as of yet, no clearly established mechanism which will accelerate particles at slow shocks. However, they have also told me that it is still an open question.

DRYER: The slow-mode waves that are associated with the slow CMEs observed by the NRL and HAO coronagraphs, must be preceded by fast-mode waves. These fast-mode waves can easily steepen in the context of a decreasing density profile in the corona. If such steepening occurs and results in shock developments (as for the not-so-slow CMEs), then particle acceleration can be expected due to gradient-B drift and/or diffusion processes.

FORBES: Yes, the compressive wave generated by a slow CME will always steepen into a fast-mode shock, even if the CME is moving at a speed less than the fast-mode wave speed. Such a fast-mode shock can produce energetic particles by the well-known diffusion process, but I am not sure if these particles would appear in time to explain Kundu's Type IV and Type II radio bursts in the lower corona.

DAVILA: Would you expect spherical geometry, i.e. additional radial gradients in B, to affect your conclusions regarding the expulsion of the filament from the solar atmosphere?

FORBES: Yes. Probably, the decrease of the magnetic field with height should help the current filament to escape, since the concentration of field near the base of the photosphere would produce a "melon-seed" effect.

UBEROI: Considering the acceleration of protons, it appears at least for auroral phenomena that Alfvén's resonance may be a better mechanism. 
FORBES: Again, it is difficult for me to say anything definite about kinetic processes within the MHD framework of my calculations. Resonance absorption could very well be important in the process.

MOGILEVSKIJ: Can such a mechanism of solar flares accelerate protons to $10^{8}-10^{10} \mathrm{eV}$ ?

FORBES: With an MHD model it is not possible to say much about the acceleration of energetic particles. However, I believe that any reconnection process which produces supermagneto-sonic outflow jets has the possibility to produce energetic protons by a Fermi-type-A-diffusion process operating at the fast-mode, termination shocks of the jets. Whether this process is quantitatively feasible, I do not yet know.

BUTI: Extending your argument about proton acceleration, could we accelerate heavier ions by this process?

FORBES: I assume so, but really I just do not know.

VAHIA: There is a lot of information that can be derived about such mechanisms, and, in particular, solar cosmic ray studies allow us to put limits on several parameters in the processes discussed here. 